\title{
Unsupervised real-time induction and interactive visualization of taxonomies over domain-specific concepts
}

\author{
$1^{\text {st }}$ Mayank Kejriwal \\ Information Sciences Institute \\ University of Southern California \\ Marina del Rey, California, United States 90292 \\ Email: kejriwal@isi.edu
}

\author{
$2^{\text {nd }}$ Ke Shen \\ Information Sciences Institute \\ University of Southern California \\ Marina del Rey, California, United States 90292 \\ Email: keshen@isi.edu
}

\begin{abstract}
Given a domain-specific set of concept labels, taxonomy induction is the problem of inducing a taxonomy over the concept labels. Despite its importance in problems such as e-commerce, and some algorithmic research as a consequence, practical tools for taxonomy induction and interactive visualization do not currently exist. To be truly useful, such a tool must permit a reasonable solution in a relatively unsupervised setting, and be applicable to general subsets of concept labels. In this paper, we present an unsupervised, end-to-end taxonomy induction system for arbitrary concept-labels from the e-commerce domain. Our system only takes a simple text file as input and yields a tree-like taxonomy that can be rendered on a browser, and that a non-technical user can interact with. Important components of the system can also be customized by a technically experienced user.
\end{abstract}

Index Terms-Taxonomy induction, e-commerce, product taxonomy, representation learning

\section{INTRODUCTION}

Taxonomies and structured representations of concepts have always played an important role in various contexts, especially in 'choice-heavy' domains such as online shopping and navigation [1], [2]. This is especially true in a digital world, when users have short attention span and are being bombarded with information and advertisements from different websites. Beyond e-commerce and Web-oriented contexts, domain-specific taxonomies play an important role in various fields and applications (e.g., scientific workflows [3]).

While in some cases, a taxonomy (or taxonomies) is 'standard' in the field and used by professionals and practitioners in that field, on e-commerce websites (including the websites for Target, Walmart and even Google Shopping), different taxonomies are in place, despite the marketplaces selling many

Permission to make digital or hard copies of all or part of this work for personal or classroom use is granted without fee provided that copies are not made or distributed for profit or commercial advantage and that copies bear this notice and the full citation on the first page. Copyrights for components of this work owned by others than ACM must be honored. Abstracting with credit is permitted. To copy otherwise, or republish, to post on servers or to redistribute to lists, requires prior specific permission and/or a fee. Request permissions from permissions@acm.org

ASONAM '21, November 8-11, 2021, Virtual Event, The Netherlands (C) 2021 Association for Computing Machinery.

ACM ISBN 978-1-4503-9128-3/21/11 ...\$15.00

http://dx.doi.org/10.1145/3487351.3489481 of the same products. On online platforms where customers make purchase decisions, a taxonomy can affect several Weboriented applications. Not only can it serve as a structured representation of the 'categories of products' (such as 'baby diapers' or 'black beans') that the platform is selling, but the taxonomy is also useful for website navigation and search [4]. A well-designed taxonomy, according to which the website's pages are linked and organized, can make for a more seamless online experience. However, the taxonomy cannot be static, since media and e-commerce companies typically have to deal with many labels (that tend to evolve as new products and topics enter the marketplace).

Given all these use-cases, automatically inducing and visualizing (with interaction) a taxonomy over a set of conceptlabels is an important and practical problem, especially for Web enterprises. In this demonstration, we present an endto-end tool that makes this task more seamless, potentially allowing non-technical domain experts and marketers to spend their time more productively while still getting the benefits of unsupervised AI tools. The input to our demonstrated system is simple: a text file that contains a set of concept-labels or 'phrases'. As we subsequently discuss, we have validated the system on real-world, widely used e-commerce conceptlabel sets for which ground-truth taxonomies are available (and hence were used for illustrating the efficacy of the system in a rigorous and quantifiable way) but we will also allow the user to input their own files into the system. Next, we use the stateof-the-art Taxonomy Induction over Concept Labels (TICL) algorithm that uses minimally supervised transfer learning techniques from the machine learning literature to induce a taxonomy over the concept-labels set without any supervision. The system does this by relying on pre-trained representation learning algorithm and background resources from the NLP and Web sciences communities, respectively. Finally, the induced taxonomy is rendered visually on a browser using opensource tools. The user can manipulate this visualization and explore the induced taxonomy. To the best of our knowledge, this is the first such openly published tool that is not only able to induce a taxonomy (without any example links) in near- 


\section{IEEE/ACM International Conference on Advances in Social Networks Analysis and Mining}

real time but also renders it in a visually appealing format for subject matter experts to navigate.

\section{RELATED WORK}

The advent of deep neural networks in the previous decade had led to significant advances in representation learning or 'embeddings' [5], [6], [7], [8], which have come to become prominent in several applied AI and machine learning domains; in particular, Natural Language Processing (NLP). Over the last five years transformer-based neural networks obtained state-of-the-art performance on several NLP tasks [6], [9], [10]. Despite being expensive to train, with many requiring ever-growing corpora and billions (and even trillions) of parameters, than previous word embedding algorithms (such as GloVE [7] and FastText [8]) needed, pre-trained versions of these algorithms have since been released in the larger community and can be 'fine-tuned' for specific application tasks and domains.

Beyond words, sentences and linguistic representations, graphs have also been embedded by similar approaches (the most recent ones of which have also been transformerbased). Examples of network embeddings include DeepWalk and node2vec [11], [12], while so-called 'knowledge graph embeddings' include RDF2vec [13], as well as popular approaches such as TransE and HolE [14]. One issue with these approaches is that they assume that the set of vertices (or what is referred to as the concept-set in this demonstration) is known in advance (before test-time); furthermore, there is an expectation of being given a set of 'positive' or example links between some of the vertices, which is then typically used to train a model and perform graph 'completion' by inferring other edges, and possibly eliminating noisy edges. Neither assumption holds here.

While our proposed system also depends on embeddings, especially in the natural language sense, it is able to work on generic e-commerce datasets with hundreds, and even thousands, of concept-labels without requiring any example edges. Furthermore, we provide an interactive visualization of the induced taxonomy. In the NLP literature, interactive visualizations (especially generated in an on-demand fashion on reasonably challenging datasets) continue to be rare, and the focus is usually on offline (or 'trained') validation on predecided benchmark datasets.

Although taxonomy (and more generally, ontology) induction has witnessed some research in domain-generic contexts, one limiting assumption is that the underlying data on which the taxonomy is induced tends to be context-rich, and often involves a set of generic nouns (e.g., WordNet extensions) [15], if not an accompanying text corpus, such as in the OntoLearn Reloaded system [16].

Algorithmic approaches best related to our system include MaxTransGraph [17], MaxTransForest [18] and TAXI [19], but none of them are specifically optimized for e-commerce data, and none can generate interactive visualizations. The system we demonstrate is easily executed as a Docker container and renders a visualization of the taxonomy with only a small lag that can be interacted with on a browser. To the best of our knowledge, this is the first openly published architecture to do so. In other work, we also showed that our underlying algorithm (TICL) outperformed these (more generic) baselines on e-commerce data.

\section{Demonstration Workflow AND USER EXPERIENCE}

We describe the anticipated user experience for the proposed demonstration. We have designed our user experience to be as seamless as possible by requiring a minimum of 'clicks' for a user to get started. This potentially allows the demonstration to be conducted virtually or over the Web, if so desired, although we are planning to conduct the demonstration on a laptop in person at the venue. $\mathrm{Ww}=\mathrm{e}$ will also allow more experienced or interested users to customize options through a web interface. The demo will be completely accessible over a browser. As described next, users will even have the ability to try the system out on their own concept-label sets.

Input: As shown in the figure, the input to the algorithm is simply a set of phrasal concept labels. The concept labels are phrasal because they may not be single words (e.g., 'beverage_service'). There is no additional information assumed to be available about them ${ }^{1}$. As described earlier, the goal is to induce a tree-like taxonomy over these concepts and produce the taxonomy in a visual form that the user can interact with. While users could input their own concept sets (via a simple text file) during the live demonstration period, we will also provide well-known publicly available examples, such as subsets of concepts from the Google Product Taxonomy $2^{2}$

TICL Algorithm: The Taxonomy Induction over Concept Labels (TICL) algorithm is a recent method developed by the authors that combines a retrofitted word embedding model from the NLP community with a graph-theoretic spanning tree algorithm to achieve good performance without seeing any training links [22], [23]. To accomplish this task robustly for arbitrary e-commerce concept-label sets, we leverage a combination of background resources, including pre-trained word embedding models [8] as well as other publicly available taxonomies on the Web for transfer learning.

Interactive Visualization: In the real world, concept-labels over which the taxonomy has to be induced can number in the hundreds or even thousands (as we showed in one of our recent papers [24]). For any induced taxonomy to be useful, the user must be able to interact with the taxonomy without getting overwhelmed. We developed the original technology assuming a general mix of both technical and non-technical users (e.g., content producers, designers and marketers). Therefore, the final output does not require complicated set up, and using D3 [25], is visualizable on a standard browser. It is also interactive; users can click on 'nodes' in the tree (specific

\footnotetext{
${ }^{1}$ This is in contrast to entities in knowledge graphs, for which additional context can be found (e.g., from Wikidata or GeoNames [20], [21]) if the entity can be linked to a canonical knowledge base, or if the knowledge graph has been constructed already from a large natural language corpus.

${ }^{2}$ https://www.google.com/basepages/producttype/taxonomy.en-US.txt
} 


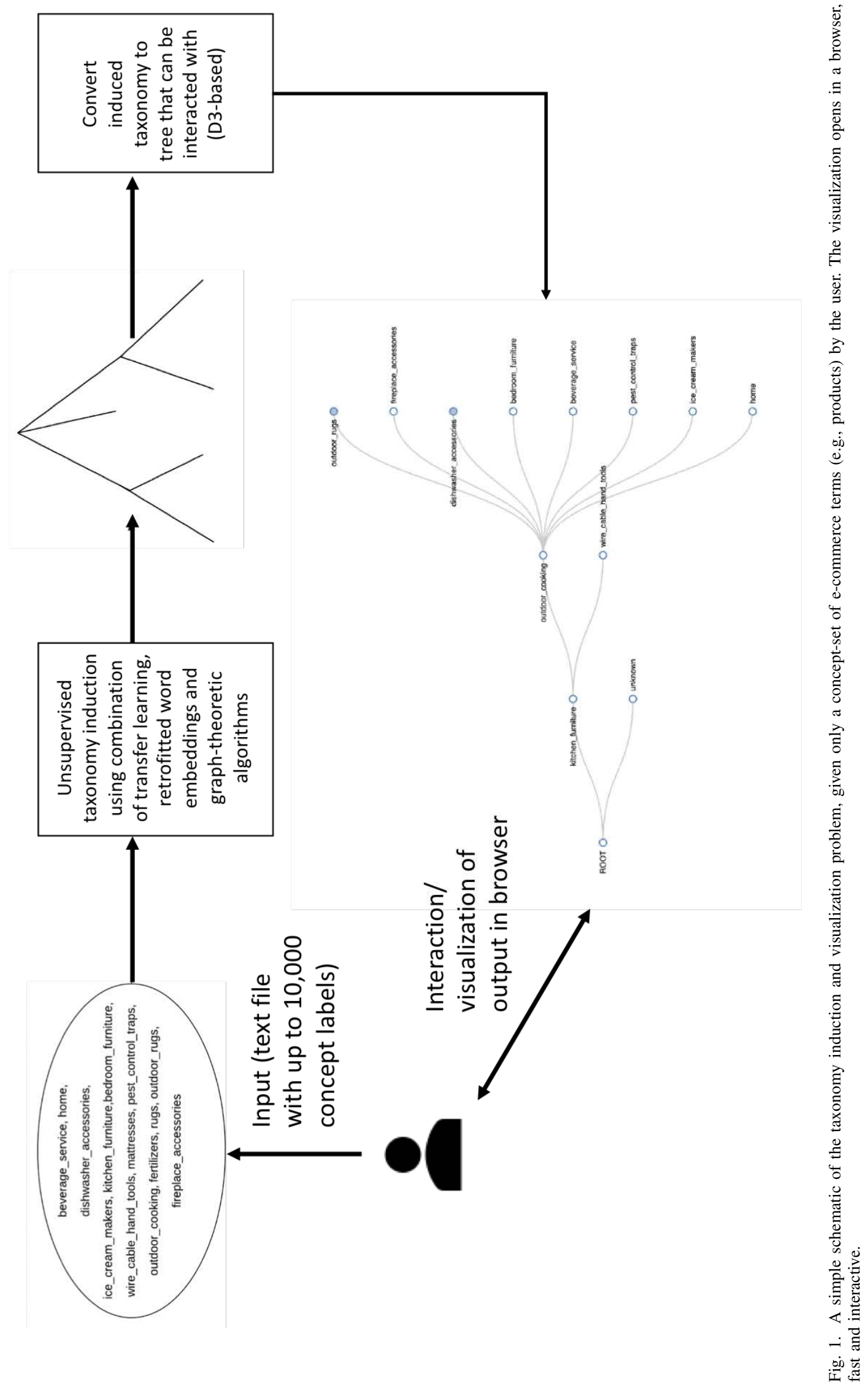




\section{IEEE/ACM International Conference on Advances in Social Networks Analysis and Mining}

concept-labels) to expand and see the set of child-nodes for that node.

In practice, this visualization is useful even for algorithmic developers, since they could tune various parameters and visualize a set of trees in different tabs on the browser to gain a more intuitive understanding of algorithmic performances and qualitative differences.

\section{SUMMARY}

Domain-specific taxonomy induction is a challenging problem with valuable applications in e-commerce and other domains with significant real-world imprint. Given concepts represented as labels, and a limited set of background resources, the problem involves inducing a taxonomy over the conceptset using the minimal inputs provided. For the underlying algorithm, we used a state-of-the-art 'transfer-based' approach called TICL. We then used open-source tools to visualize the generated taxonomy in an interactive fashion in a browser. In the demonstration, we will allow users to upload their own concept-sets and interact with the taxonomy, and we will also provide real-world e-commerce data for potential users to work with.

\section{ACKNOWLEDGMENT}

The authors would like to thank Nicolas Torzec and ChienChun Ni from the Yahoo! Knowledge Graph Research Group for helping us with the data and visualization code, which is based on open-source packages. This project was primarily funded under a Yahoo! Faculty Research Engagement Program grant awarded to Kejriwal.

\section{REFERENCES}

[1] Y. S. Kim, "Recommender system based on product taxonomy in e-commerce sites," Journal of information science and engineering, vol. 29, no. 1, pp. 63-78, 2013.

[2] H. Davulcu, S. Koduri, and S. Nagarajan, "Datarover: a taxonomy based crawler for automated data extraction from data-intensive websites," in Proceedings of the 5th ACM international workshop on Web information and data management, 2003, pp. 9-14.

[3] J. Yu and R. Buyya, "A taxonomy of scientific workflow systems for grid computing," ACM Sigmod Record, vol. 34, no. 3, pp. 44-49, 2005.

[4] M. N. Uddin and P. Janecek, "Performance and usability testing of multidimensional taxonomy in web site search and navigation," Performance measurement and metrics, 2007.

[5] T. Mikolov, I. Sutskever, K. Chen, G. S. Corrado, and J. Dean, "Distributed representations of words and phrases and their compositionality," in Advances in neural information processing systems, 2013, pp. 3111-3119.

[6] J. Devlin, M.-W. Chang, K. Lee, and K. Toutanova, "Bert: Pre-training of deep bidirectional transformers for language understanding," arXiv preprint arXiv:1810.04805, 2018.

[7] J. Pennington, R. Socher, and C. D. Manning, "Glove: Global vectors for word representation," in Proceedings of the 2014 conference on empirical methods in natural language processing (EMNLP), 2014, pp. $1532-1543$.

[8] A. Joulin, E. Grave, P. Bojanowski, and T. Mikolov, "Bag of tricks for efficient text classification," arXiv preprint arXiv:1607.01759, 2016.

[9] Y. Liu, M. Ott, N. Goyal, J. Du, M. Joshi, D. Chen, O. Levy, M. Lewis, L. Zettlemoyer, and V. Stoyanov, "Roberta: A robustly optimized bert pretraining approach," arXiv preprint arXiv:1907.11692, 2019.

[10] T. B. Brown, B. Mann, N. Ryder, M. Subbiah, J. Kaplan, P. Dhariwal, A. Neelakantan, P. Shyam, G. Sastry, A. Askell et al., "Language models are few-shot learners," arXiv preprint arXiv:2005.14165, 2020.
[11] B. Perozzi, R. Al-Rfou, and S. Skiena, "Deepwalk: Online learning of social representations," in Proceedings of the 20th ACM SIGKDD international conference on Knowledge discovery and data mining, 2014, pp. 701-710.

[12] A. Grover and J. Leskovec, "node2vec: Scalable feature learning for networks," in Proceedings of the 22nd ACM SIGKDD international conference on Knowledge discovery and data mining, 2016, pp. 855864.

[13] P. Ristoski and H. Paulheim, "Rdf2vec: Rdf graph embeddings for data mining," in International Semantic Web Conference. Springer, 2016, pp. $498-514$

[14] M. Kejriwal, "Advanced topic: Knowledge graph completion," in Domain-Specific Knowledge Graph Construction. Springer, 2019, pp. $59-74$.

[15] R. Snow, D. Jurafsky, and A. Y. Ng, "Semantic taxonomy induction from heterogenous evidence," in Proceedings of the 21st International Conference on Computational Linguistics and the 44th annual meeting of the Association for Computational Linguistics. Association for Computational Linguistics, 2006, pp. 801-808.

[16] P. Velardi, S. Faralli, and R. Navigli, "Ontolearn reloaded: A graph-based algorithm for taxonomy induction," Computational Linguistics, vol. 39, no. 3, pp. 665-707, 2013.

[17] J. Berant, I. Dagan, and J. Goldberger, "Learning entailment relations by global graph structure optimization," Computational Linguistics, vol. 38, no. 1, pp. 73-111, 2012. [Online]. Available: https://www.aclweb.org/anthology/J12-1003

[18] J. Berant, N. Alon, I. Dagan, and J. Goldberger, "Efficient global learning of entailment graphs," Computational Linguistics, vol. 41, no. 2, pp. 249-291, 2015. [Online]. Available: https: //doi.org/10.1162/COLI_a_00220

[19] A. Panchenko, S. Faralli, E. Ruppert, S. Remus, H. Naets, C. Fairon, S. P. Ponzetto, and C. Biemann, "Taxi at semeval-2016 task 13: a taxonomy induction method based on lexico-syntactic patterns, substrings and focused crawling," in Proceedings of the 10th International Workshop on Semantic Evaluation. San Diego, CA, USA: Association for Computational Linguistics, 2016.

[20] D. Vrandečić and M. Krötzsch, "Wikidata: a free collaborative knowledgebase," Communications of the ACM, vol. 57 , no. 10 , pp. 78-85, 2014.

[21] M. Kejriwal and P. Szekely, "Neural embeddings for populated geonames locations," in International Semantic Web Conference. Springer, 2017, pp. 139-146.

[22] M. Faruqui, J. Dodge, S. K. Jauhar, C. Dyer, E. Hovy, and N. A. Smith, "Retrofitting word vectors to semantic lexicons," arXiv preprint arXiv:1411.4166, 2014.

[23] J. B. Kruskal, "On the shortest spanning subtree of a graph and the traveling salesman problem," Proceedings of the American Mathematical society, vol. 7, no. 1, pp. 48-50, 1956.

[24] M. Kejriwal, R. K. Selvam, C.-C. Ni, and N. Torzec, "Locally constructing product taxonomies from scratch using representation learning."

[25] S. Murray, Interactive data visualization for the web: an introduction to designing with D3. " O'Reilly Media, Inc.", 2017. 